\title{
Comparison of dissection speed during colorectal ESD between the novel Multiloop (M-loop) traction method and ESD methods without traction $\square$
}

\section{(ㄷ)(우우}

\author{
Authors \\ Nakase $^{2}$ \\ Institutions \\ 1 Center for gastroenterology, Teine Keijinkai Hospital, \\ Sapporo, Japan \\ 2 Department of Gastroenterology and Hepatology, \\ Sapporo Medical University School of Medicine, \\ Sapporo, Japan. \\ 3 Center for Translational Research, The institute of \\ Medical Science Hospital, The University of Tokyo, \\ Japan.
}

Yuichiro Suzuki ${ }^{1}$, Tokuma Tanuma ${ }^{1}$, Masanori Nojima ${ }^{3}$, Gota Sudo ${ }^{1,2}$, Yuki Murakami ${ }^{1}$, Tatsuya Ishii ${ }^{1}$, Masakazu Akahonai $^{1}$, Yosuke Kobayashi ${ }^{1}$, Hidetaka Hamamoto ${ }^{1}$, Hironori Aoki ${ }^{1}$, Taku Harada ${ }^{1}$, Akio Katanuma ${ }^{1}$, Hiroshi

submitted 3.11 .2019

accepted after revision 30.3.2020

\author{
Bibliography \\ DOI https://doi.org/10.1055/a-1161-8596 | \\ Endoscopy International Open 2020; 08: E840-E847 \\ (c) Georg Thieme Verlag KG Stuttgart · New York \\ eISSN 2196-9736
}

Corresponding author

Yuichiro Suzuki, MD, Center for Gastroenterology, Teine Keijinkai Hospital, 1-40-12-1, Maeda, Teine-ku, Sapporo 006-8555, Japan

Fax: +81-11-685-2998

yuichiro.suzuki.kpc@gmail.com

\section{ABSTRACT}

Background and study aims We previously reported on a novel traction method called Multiloop (M-loop) for faster colorectal endoscopic submucosal dissection (ESD). In this study, we retrospectively compared the difference in sub- mucosal dissection time (SDT), and submucosal dissection speed (SDS) between groups of patients who were treated using traction with the M-loop method, and with non-traction methods of colorectal ESD.

Patients and methods We reviewed and timed duration of colorectal ESD by the non-traction method from videos recorded between June 2016 and December 2017. From January 2018 onward, we used the M-loop method during all colorectal ESDs and timed it until August 2018. Outcomes of colorectal ESD with the M-loop method and nontraction methods were compared. The study involved two experts and eight non-experts and was carried out at a tertiary endoscopic center in Japan.

Results The study included 50 patients who treated with the M-loop method and 115 patients treated with the nontraction method. Submucosal dissection time (SDT) was not significantly different (M-loop group, $42.1 \pm 4.2 \mathrm{~min}$, non-traction ESD group, $51.9 \pm 3.3 \mathrm{~min})(P=0.098)$, but submucosal dissection speed (SDS) was significantly greater (M-loop group, $28.0 \pm 2.9 \mathrm{~mm}^{2} / \mathrm{min}$, non-traction ESD group, $\left.19.9 \pm 2.0 \mathrm{~mm}^{2} / \mathrm{min}\right)(P=0.0014)$ in the $M$-loop method group. Multivariate analysis showed that the M-loop method increased SDS by odds ratio of 1.46 $(P=0.001)$ when compared to the non-traction ESD method. A significant difference was also observed for SDT and SDS when the two methods were compared after propensity score matching $(P=0.001)$. No differences in unfavorable outcomes were observed.

Conclusions The M-loop method improved SDS compared to non-traction methods of ESD. The method is an effective tool to assist colorectal ESD.

\section{Introduction}

Endoscopic submucosal dissection (ESD) is an effective method of treating large superficial colorectal neoplasms because it results in increased curative resection rates compared to endoscopic mucosal resection (EMR) [1,2]. The clinical issue is the longer procedure time, which makes standardization of colorectal ESD more difficult. Methods to create traction have been developed as a way of overcoming this difficulty $[3,4]$. Traction creates tension in the submucosal plane, allowing quicker identification of line of dissection and blood vessels. Because of this property, traction can speed up colorectal ESD 


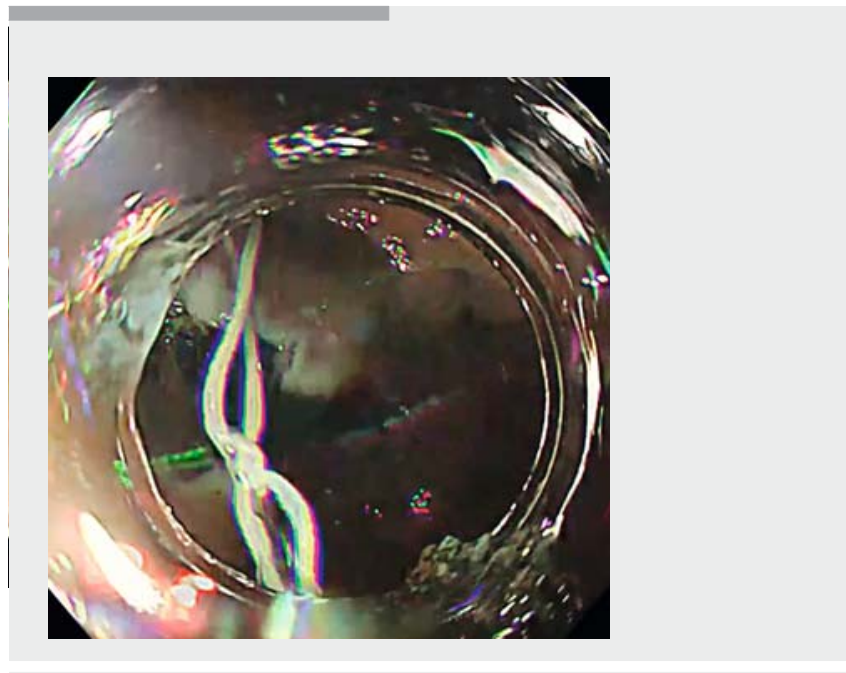

$\checkmark$ Video 1 The M-loop is tied around a 2.5-cc syringe twice, then the redundant thread is trimmed. M-loop is two loops are stored for later use. The use of M-loop during colorectal ESD. After circumferential excision is made, the M-loop is attached onto the edge of the lesion. The second, free end of the loop is attached to the contralateral wall with an extra clip, which gives great visualization and counter-traction of submucosal tissue. The thread of the M-loop is dissected after the lesion is dissected off of the wall, and the specimen is collected.

and reduce the burden on both patient and physician. Several techniques for traction, which include clip with line method [3], clip and snare method [5], and S-O clip method, [6] have been reported. These methods facilitate dissection, but they either require additional maneuvers such as reinsertion of the scope, or require costly adjunct devices that are not available in some institutions. On the other hand, a non-traction method, the pocket-creation method (PCM) has been invented by Hayashi et al [7]. It involves creation of a submucosal pocket into which the tip of the endoscope is inserted to directly visualize the submucosa. PCM requires neither reinsertion of the scope nor adjunct devices. It stabilizes the view of the submucosal dissection plane, and reportedly is associated with fewer adverse events (AEs) than the conventional method of colorectal ESD [8]. However, PCM is still difficult for many non-experts.

To facilitate colorectal ESD by ESD non-experts, we previously reported use of a more accessible and cost-effective traction technique, called the multiloop (M-loop) method, which can done with only surgical sutures and a clip $[9,10]$. In this retrospective observational study, we investigated the dissection speed of the M-loop method for colorectal ESD in comparison with non-traction methods including PCM and the conventional method.

\section{Patients and methods}

\section{Patients}

All ESDs were planned according to Japanese guidelines for ESD and EMR of colorectal cancer [11]. Antithrombotic agents were stopped prior to ESD according to the current guideline [12].
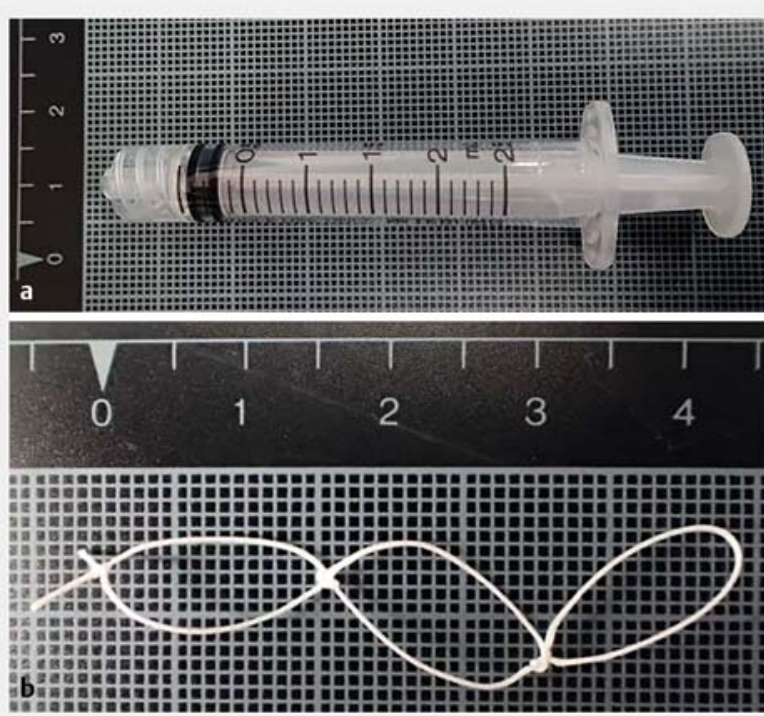

- Fig. 1 a The surgical suture is tied around this $2.5 \mathrm{cc}$ syringe to create M-loop. b M-loop with triple loops is made, which is stored for later use.

Data from patients who were treated by non-traction methods of colorectal ESD from June 2016 until December 2017 were retrieved from an electronic database, and procedural video was retrospectively reviewed to time ESD duration. Beginning in January 2018, the M-loop method was used to treat all cases of colorectal ESD, and from that point onward, procedure times for all ESDs were recorded until August 2018. Duration of ESD was compared between the two groups. All informed consent processes for this study were conducted in accordance with guidelines of the Teine Keijinkai Hospital Institutional Review Board.

\section{M-loop design}

M-loop was created and used in the same manner as previously reported $[9,10]$. A 3-0 silk suture thread (Sterilized Matsuda Suture; Matsuda) was reeled once around a 2.5-cc syringe and a knot was made. We then reeled the thread back around the syringe to tie a second knot, and the redundant thread was trimmed ( $\vee$ Video $\mathbf{1}$ ). By repeating this process, the number of loops can be adjusted, depending on the diameter of the colonic lumen. For example, $\boldsymbol{\nabla}$ Fig. $\mathbf{1} \mathbf{a}$ and $\boldsymbol{\nabla}$ Fig. $\mathbf{1} \mathbf{b}$ show an M-loop with triple loops. The loop was stored for later use for ESD. The loop was attached onto a clip and housed within the delivery sheath before its use.

Actual use of M-loop during colorectal ESD is shown in -Video 1. The M-loop is introduced into the colorectal lumen and attached to the edge of the lesion. The free end of the loop is then clipped onto the contralateral wall using another clip. Submucosal tissue is clearly visualized with excellent counter traction, and dissection is continued. The thread of the $\mathrm{M}$-loop is cut with a hook knife, and the specimen is collected. 


\section{ESD procedure}

All patients were given sodium picosulfate hydrate the night before the procedure and instructed to take polyethylene glycol mixed with dimethicone on the day of the procedure. Patients were sedated using intravenous (IV) midazolam or propofol. IV pethidine was used for analgesia on an as-needed basis. Colorectal ESD was performed using a single-channel endoscope with jet function (PCF-H290AZI; Olympus Medical Systems, Tokyo, Japan). A transparent short hood was attached to the endoscope tip (Elastic touch, L, 16675; TOP co, Tokyo, Japan, or ST hood short type; Fujifilm, DH-30CR for PCM).

We performed colorectal ESD with non-traction methods (conventional ESD and PCM) from 2016 until 2018. Hook knife (KD-620LR; Olympus Medical Systems, Tokyo, Japan) was used for all cases of colorectal ESD. ESD with PCM was undertaken in a similar manner as previously reported [13], and so was conventional ESD [12]. As for PCM, we used a short type ST hood rather than a conventional ST hood, and a hook knife was used instead of needle-type knives. Because there was not enough space within the hood, the hook knife was rotated outside the hood. For the above reasons, strictly speaking, our PCM is more a modified PCM. As for the electrosurgical unit (ESU), we used a VIO 300 D until 2017, and then switched to VIO 3 at the beginning of 2018. The settings are shown in $>$ Table 1 . Sodium hyaluronate solution mixed with indigo carmine was injected to lift the lesion. As with the method previously described, we attached the M-loop to an opening-and-closing clip (EZ Clip, Olympus Medical Systems, Tokyo, Japan), which was introduced into the colorectal lumen via the working channel. The $\mathrm{M}$-loop was clipped onto the edge of the exfoliated mucosa right after circumferential incision. We then introduced another clip, which was used to attach the free end of the loop to the contralateral wall. As a result, we obtained excellent traction and visualization of the submucosal layer [9]. During dissection of larger lesions, the traction sometimes become slack and insufficient. In such cases, a new clip was hooked onto the knot in between the loops and reattached onto the contralateral wall. This brought the lesion closer to the contralateral wall, which strengthened the traction to better expose the submu-
Table 1 Electro-surgical unit (ESU) settings during the study period.

\begin{tabular}{|l|l|l|}
\hline & Until 2017 & From 2018 \\
\hline & VIO300 D & VIO3 \\
\hline Marking & Forced coag. E3 30 W & Forced coag. E3.5 \\
\hline Mucosal incision & EndoCut I E2 D3 I3 & EndoCut I E2 D3 I3 \\
\hline Dissection & Swift coag. E3 40W & Swift coag. E3.5 \\
\hline Hemostasis & Spray coag. E2 40W & Spray coag. E2.5 \\
\hline (Coagrasper) & Soft coag E5 80W & Soft coag E6.5 \\
\hline E, effect; D, duration; I, interval & \\
\hline
\end{tabular}

cosa ( $\vee$ Fig. 2a, $\triangleright$ Fig. 2b, $\triangleright$ Fig. 2c, $\triangleright$ Fig. 2 d). After completion of dissection, the loop was cut with the same electrosurgical knife used for dissection. The tissue was sent for pathological analysis by an experienced pathologist independent of the endoscopists.

\section{Outcomes}

As the primary outcome of this study, we retrospectively compared average submucosal dissection speed (SDS) for the Mloop method and non-traction methods of ESD by PCM, and the conventional method of ESD.

Secondary outcomes were total procedure time, R0 resection rate, curative resection rate, and rates of perforation and delayed bleeding for the M-loop versus the non-traction group. We also investigated how the method of dissection influenced outcomes between experts and non-experts. Multivariate analysis was conducted to examine the effect of the M-loop method on SDS adjusted for potential confounding factors and to extract factors that might have influenced the outcome.

\section{Definitions}

Patients who were treated by conventional ESD or PCM was categorized as the non-traction group. SDT was then calculated for each method. For PCM, we defined SDT as the time from insertion of the endoscope into the submucosal layer to comple-

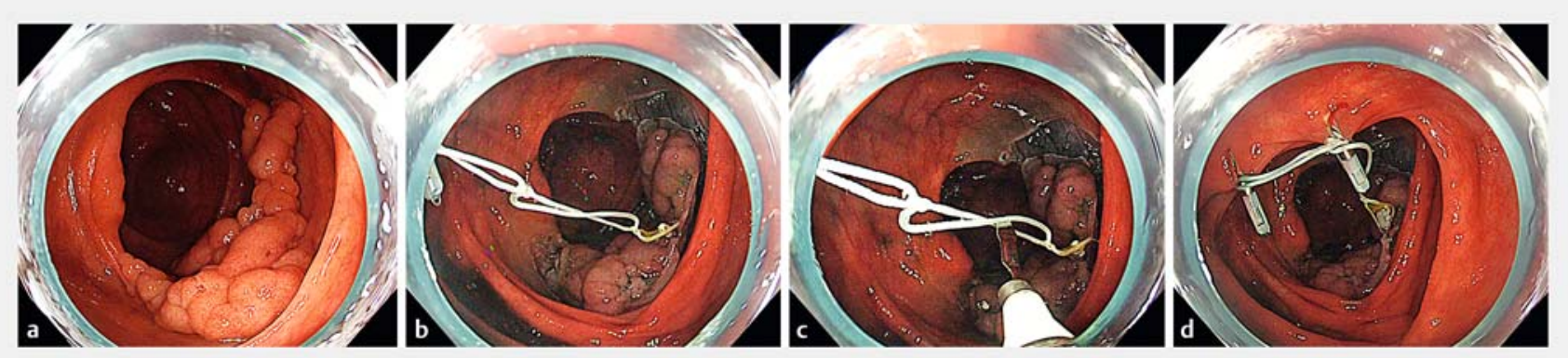

- Fig. 2 Strengthening of traction during colorectal ESD. a LST-G $70 \mathrm{~mm}$ in size was located in the cecum down to ascending colon. $\mathbf{b} \mathrm{M}$-loop with three loops (white thread) are attached to the lesion and contralateral wall with clips. c, d A new clip was introduced via working channel, and hooked onto the second loop and attached to contralateral wall for intensified traction, which gave excellent traction that effectively exposed submucosal layer. 
tion of tumor dissection. We defined SDT for the conventional ESD method as the time from completion of circumferential dissection to completion of dissection. As for the M-loop method, SDT was defined as time from the creation of first traction of the M-loop to completion of tumor dissection. We defined the time taken for M-loop attachment as time from introduction and opening of the M-loop-attached clip in the colon to successful attachment of the M-loop onto the contralateral wall with another clip. SDT was defined as time from creation of first traction with the M-loop until completion of tumor dissection. We defined total procedure time as the time from when the first injection was made until completion of dissection.

The specimen area was calculated by multiplying length and width of the specimen. We calculated SDS by dividing the area of the resected specimen by SDT.

$\mathrm{R} 0$ resection was defined as one-piece resection of the tumor with free margins. Curative resection was defined as intramucosal tumor or slightly invasive $(<1000 \mu \mathrm{m})$ submucosal cancer having free horizontal and vertical margins, and no lymphovascular invasions. Submucosal fibrosis was determined visually and grouped according to categories proposed by Matsumoto et al., which is classification by presence and extent of white thread directly after submucosal injection [14].

An expert endoscopist was defined as an operator who performed more than 80 colorectal ESD cases, and non-expert was defined as someone who performed fewer than 80 colorectal ESD cases before this study [15]. This study involved two experts and eight non-experts, and their case experience is shown on $>$ Table 2 . Ten, 17 , and 14 cases in the expert group were treated with the M-loop method, PCM method, and conventional method, respectively. Forty, 62, and 22 cases in the nonexpert group were treated with the M-loop method, PCM, and conventional method, respectively. In principle, a non-expert was supervised by expert endoscopists who had taken over the procedure in difficult cases of colorectal ESD.

To assess unfavorable outcomes efficiently, we defined a case with an unfavorable outcome as a patient in whom at least one of the following occurred: non-R0 resection, non-curative resection, perforation, or delayed bleeding. Perforation was defined as a full-thickness defect of the colorectal wall, which was recognized by the operator as a state in which connective tissue, adipose tissue, and/or serosa was visible through the defect. Delayed bleeding was defined as presence of marked bloody stool after treatment or a requirement for hemostasis after treatment [12].

\section{Statistical analysis}

We used a chi-squared test for comparison of categorical variables. For comparison of continuous variables, a Mann Whitney $U$ test was used.

Multivariate analysis using multiple linear regression was conducted to examine the effect of the M-loop method on SDS adjusted for potential confounding factors (age, sex, location, area, macroscopic type, the presence of fibrosis, and operator's experience) and to extract factors that may have an influenced the outcome. Because distribution of the SDS was lognormal distribution, we log-transformed the value for the mod-
- Table 2 Number of colorectal ESD cases performed per endoscopist at the beginning of June 2016 and January 2018.

\begin{tabular}{|l|c|c|}
\hline & Jun-16 & Jan-18 \\
\hline Expert 1 & 212 & 348 \\
\hline Expert 2 & 41 & 156 \\
\hline Non-expert 1 & 17 & 76 \\
\hline Non-expert 2 & 6 & 26 \\
\hline Non-expert 3 & 7 & 13 \\
\hline Non-expert 4 & 3 & 40 \\
\hline Non-expert 5 & 0 & 15 \\
\hline Non-expert 6 & 2 & 9 \\
\hline Non-expert 7 & 0 & 0 \\
\hline Non-expert 8 & 0 & 0 \\
\hline
\end{tabular}

el and then the estimates could be interpreted as the ratio of the speed. An explanatory variable with log-normal distribution was also log-transformed. Continuous variables were basically included in the model without categorization unless non-linear correlation and severe skewness were observed. As a sensitivity analysis, we performed a comparison with propensity score matching (nearest neighbor matching with caliper $<0.1$ ). Propensity score for the method selection was calculated using logistic regression model with the potential confounders described above.

SPSS 23 (IB, New York, United States) was used for all statistical analyses and $P<0.05$ was considered statistically significant.

\section{Results}

A total of 225 patients with 236 lesions were treated with colorectal ESD between June 2016 and August 2018 at Teine Keijinkai hospital, a tertiary endoscopic center. The following cases were excluded from the study: 10 cases for missing video, one case because ESD was aborted due to deep submucosal invasion, 11 cases because EMR was done at the same time as ESD to avoid obscurity of the video, and 38 cases because other methods of traction were used (such as S-O clip). When multiple lesions weree dissected in one specimen, the lesion was counted as one. After exclusion, 165 patients with 165 lesions were included in the study, of whom 50 and 115 patients were treated by ESD with the M-loop method, and non-traction methods (36 patients by conventional ESD method, 79 patients by $\mathrm{PCM}$ ), respectively ( $\mathbf{F i g . 3}$ ). All patients in the M-loop group are the same group of patients included in our previously published article on the M-loop method [10]. Eighty percent of the operators participating in this study were non-experts. Eight cases of ESD by non-expert endoscopists required assistance of an expert endoscopist, but other cases were completed independently. 
June 2016 - December 2017

175 cases and 187 lesions of colorectal ESD (PCM + conventional methods)

August 2018

50 cases of colorectal

ESD with 50 lesions

(M-loop method)

- 165 cases of colorectal ESD with 165 lesions

- 50 cases treated by M-loop method

- 115 cases treated by non-traction methods

- (79 cases by PCM, 36 cases by conventional ESD)

Fig. 3 Selection flow chart of the patients included in this study.

Patient characteristics are shown in $>$ Table 3 . Five of 50 lesions and 26 of 115 lesions were located in the rectum in the $\mathrm{M-}$ loop group and non-traction group, respectively. We tumor represented recurrence after endoscopic treatment nor were any of the tumors biopsied prior to treatment. There were no notable differences between the groups.
As for attachment of the M-loop, mean time to successful clip attachment was 2 minutes, 54 seconds. The success rate was $98 \%$ because of failure in one case as a result of loosening of the loop. The average number of M-loops and EZ clips used were 1.24 and 2.62, respectively. $>$ Table 4 lists the primary and secondary outcomes for the two groups. The M-loop method had a significantly greater SDS compared to non-traction methods (M-loop method group, $28 \pm 2.9 \mathrm{~mm}^{2} / \mathrm{min}$, non-traction ESD group, $\left.19.9 \pm 2.0 \mathrm{~mm}^{2} / \mathrm{min}\right)(P=0.0014)$. SDT of $\mathrm{M}$ loop was not statistically significant when compared to the non-traction method (M-loop method group, $42.1 \pm 4.2 \mathrm{~min}$ utes, non-traction ESD group, $51.9 \pm 3.3$ minutes) $(P=0.098)$. With regard to secondary outcomes, no significant difference was observed in total procedural time and in unfavorable outcomes including both technical and oncological outcomes between the two groups. - Table 5 shows differences of average SDS between the two groups for both experts and non-experts. In the expert group, the mean SDS was greater with the M-loop method than with non-traction methods with no significant difference. However, a significant difference in SDS was observed in non-experts (M-loop group, $25.3 \pm 3.1 \mathrm{~mm}^{2} / \mathrm{min}$, non-traction group, $16.5 \pm 2.4)(P=0.001)$.

Multivariate analysis using multiple linear regression analysis showed that use of the M-loop method made colorectal ESD faster by an odds ratio of 1.46 compared to the non-traction method $(P=0.0014)$. Dissection speeds for experts were significantly higher than for non-experts by an odds ratio of $1.85(P<$ $0.001)$. Colorectal ESD tended to be faster for larger lesions

- Table 3 Comparison of patient and lesion characteristics between M-loop group and non-traction group.

\begin{tabular}{|c|c|c|c|c|}
\hline & & M-loop $(n=50)$ & Non-traction $(n=115)$ & $P$ value \\
\hline \multirow[t]{2}{*}{ Age } & Mean & 71.5 & 69.2 & 0.069 \\
\hline & Median & 74 & 70 & \\
\hline \multirow[t]{2}{*}{ Gender } & M & 36 & 63 & 0.149 \\
\hline & $\mathrm{F}$ & 14 & 52 & \\
\hline \multirow[t]{3}{*}{ Location } & Right colon & 33 & 61 & 0.128 \\
\hline & Left colon & 12 & 28 & \\
\hline & Rectum & 5 & 26 & \\
\hline \multirow[t]{3}{*}{ Fibrosis } & F0 & 40 & 99 & 0.602 \\
\hline & F1 & 8 & 12 & \\
\hline & $\mathrm{F} 2$ & 2 & 4 & \\
\hline \multirow[t]{2}{*}{ Specimen size } & Mean & 873.2 & 786.9 & 0.149 \\
\hline & Median & 760.7 & 628 & \\
\hline \multirow[t]{2}{*}{ Operator } & Expert & 10 & 31 & 0.434 \\
\hline & Non-expert & 40 & 84 & \\
\hline \multirow[t]{2}{*}{ Morphology } & Flat & 44 & 88 & 0.137 \\
\hline & Elevated & 6 & 27 & \\
\hline Previous biopsy & & 0 & 0 & NA \\
\hline Recurrent lesion & & 0 & 0 & NA \\
\hline
\end{tabular}


Table4 Differences in outcomes between M-loop group and non-traction group.

\begin{tabular}{|l|l|l|l|}
\hline & & M-loop (n= 50) & Non-traction (n=115) \\
\hline Total procedure time, min & Mean \pm SE & $69.2 \pm 4.9$ & $64.5 \pm 3.4$ \\
\hline & Median & 57 & 56 \\
\hline Submucosal dissection time, min & Mean \pm SE & $42.1 \pm 4.2$ & $51.9 \pm 3.3$ \\
\hline & Median & 32 & 45 \\
\hline Submucosal dissection speed, $\mathrm{mm}^{2} / \mathrm{min}$ & Mean \pm SE & $28.0 \pm 2.9$ & $19.9 \pm 2.0$ \\
\hline & Median & 21.4 & 15.7 \\
\hline R0 resection, $\mathrm{n}(\%)$ & & $50(100 \%)$ & $115(100 \%)$ \\
\hline Curative resection, $\mathrm{n}(\%)$ & & $48(96 \%)$ & $106(92 \%)$ \\
\hline Perforation, $\mathrm{n}(\%)$ & & $2(4 \%)$ & 6.098 \\
\hline Delayed bleeding, $\mathrm{n}(\%)$ & $0(0 \%)$ & $2(1.7 \%)$ \\
\hline Undesired outcome, $\mathrm{n}(\%)$ & $3(6.0 \%)$ & $19(16.5 \%)$ \\
\hline
\end{tabular}

- Table 5 Differences in average SDS $\left(\mathrm{mm}^{2} / \mathrm{min}\right)$ between the M-loop and non-traction groups in experts and non-experts.

\begin{tabular}{|l|l|l|l|l|}
\hline Operator & & M-loop $(\mathbf{n}=\mathbf{1 0})$ & Non-traction $(\mathbf{n}=\mathbf{3 1})$ & $\mathbf{P}$ value \\
\hline Expert & mean & $38.9 \pm 6.9$ & $29.1 \pm 3.2$ & 0.099 \\
\hline & median & 33.8 & 21.8 \\
\hline & & Multiloop $(\mathbf{n}=40)$ & Non-traction $(\mathbf{n}=84)$ \\
\hline Non-expert & mean & $25.3 \pm 3.1$ & $16.5 \pm 2.4$ & 0.001 \\
\hline & median & 19.5 & 13.3 \\
\hline M-loop, multi-loop & & & \\
\hline
\end{tabular}

(1.36 per 2 times area increase, $P<0.001)$, when patients were younger (0.99 per 1-year increase, $P=0.021)$, and when patients were male $(1.21, P=0.037)$ ( $\triangleright$ Table 6$)$. We also performed a comparison with propensity score matching as a sensitivity analysis (48 cases per group, total 96 cases, were matched), and a similar result was observed; median of SDS was 24.1 (interquartile range: 14.4-36.2) for M-loop, and 14.2 $(11-17.5)$ for the non-traction method $(P=0.001)$. Median SDT for the M-loop was significantly shorter at 32 (21.3-51) when compared to 56.5 (37-72.5) for non-traction methods $(P=0.001)$. There was no difference in total procedure time when the two groups were compared with the propensity score matching; median 56.5 (44.0-91.5) for M-loop and 73.5 (51.093.0) for the non-traction method $(P=0.187)$.

\section{Discussion}

This study demonstrated that the M-loop method for colorectal ESD can help endoscopists achieve greater SDS than non-traction methods. Of note, SDS with use of the M-loop in non-experts was significantly increased in comparison to non-traction methods of ESD, and although not statistically significant, the M-loop method increased SDS even when used by experts. After propensity score analysis, the SDT was significantly short- er, and SDS significantly greater for the M-loop method, although total procedure time for the M-loop method was not significantly different from that for the non-traction method. Unfavorable outcome associated with M-loop method was not observed. Taking all of this information together, we consider this new traction method as an attractive new therapeutic approach for colorectal ESD.

ESD helps patients with large superficial colorectal neoplasms avoid surgery. On the other hand, variation in size and location of the polyps in the colon determines how difficult it will be to perform colorectal ESD. Therefore, many methods and devices have been developed to overcome difficult cases. PCM has advantages over conventional methods of ESD because it helps stabilize the view when the endoscope is inserted into the pocket, spontaneously adjusting the scope, and it is effective even when there is extensive submucosal fibrosis [16]. Even though in a previous study, PCM was not shown to increase SDS when compared to conventional ESD [8], its advantage of PCM is that neither adjunct devices nor complicated maneuvers are required for safe execution, unlike most ESD traction methods. On the other hand, PCM requires making a submucosal pocket, which can be a technical hurdle, especially for non-experts. Therefore, PCM may not be suitable for ESD 
- Table6 Multivariate analysis using linear regression analysis for the factors associated with SDS.

\begin{tabular}{|l|l|l|l|l|}
\hline & & & $95 \%$ Confidence Interval \\
\hline Parameter & P value & Ratio of the speed & Lower Bound \\
\hline M-loop vs. non-traction & 0.001 & 1.46 & 1.15 & 1.70 \\
\hline Age (per 1-year increase) & 0.021 & 0.99 & 0.98 & 1.00 \\
\hline Male vs. Female & 0.037 & 1.21 & 1.01 & 1.45 \\
\hline Right vs. Left & 0.529 & 1.06 & 0.89 & 1.27 \\
\hline Area (per 2 times increase) & $<0.001$ & 1.36 & 0.83 & 1.50 \\
\hline Elevated vs. Flat & 0.651 & 1.06 & 0.65 \\
\hline Fibrosis (per 1-category increase) & 0.015 & 0.78 & 1.50 \\
\hline Expert vs. Non-expert & $<0.001$ & 1.85 & 0.95 \\
\hline
\end{tabular}

training and other feasible methods should be used in that setting.

As we already have reported $[9,10]$, the M-loop can be made easily in a few minutes with readily available surgical thread and clip. Our data indicate that attachment time to the lesion with the M-loop (approximately 2 minutes, 54 seconds) was similar to that with S-O clip for colorectal lesions (2 minutes). Importantly, our data suggest that the M-loop method contributed to improved endoscopic performance for both non-experts and experts, based on the SDS data.

Generally, identifying the plane of dissection during colorectal ESD is most difficult for novice endoscopists. Based on our data demonstrating a significant improvement in SDS with use of the M-loop method by non-experts, we consider it a device useful for assistance and for training for non-experts in colorectal ESD.

When compared to other traction methods, the M-loop has advantages as follows: (1) no necessity for reinserting the scope; (2) application in segments with large lumen, such as the cecum and rectum, by adding loops; and (3) low cost. For example, the cost of one S-O clip is 45 USD (5000 JPN yen), while the cost of making three M-loops, which requires one 3-
0 thread, is about 20 cents (23.3 JPN yen), and two clips (EZ clip, Olympus Medical System), which are about 9 USD (970 JPN yen) each. As shown in this study, the average number of M-loops and EZ clips used were 1.24 and 2.62, respectively, and therefore the average total cost would be about 23.90 USD (about 2600 JPN). Because of its low cost, an extra loop can be added for traction in different segments of the exfoliated lesion with little concern about added expense ( $\triangleright$ Fig. $\mathbf{4 a}$, > Fig. 4b, > Fig. 4c, D Fig. 4d). Thus, the simplicity and cost-effectiveness of the M-loop method makes it a versatile tool in different ESD scenarios.

Multivariate analysis showed slower SDS for lesions with more fibrosis (ratio of the speed $0.81, P=0.041$ ), which was consistent with previous reports $[14,17,18]$. We found that the average SDS increased for experts, and, unexpectedly, it became greater with increasing specimen size. These data strongly suggest the advantage of the M-loop for enabling both expert and non-expert operators to easily perform colorectal ESD by stabilizing the view of the submucosal dissection plane.

Because significantly greater SDS and shorter SDT for Mloop were observed after propensity score matching, the total
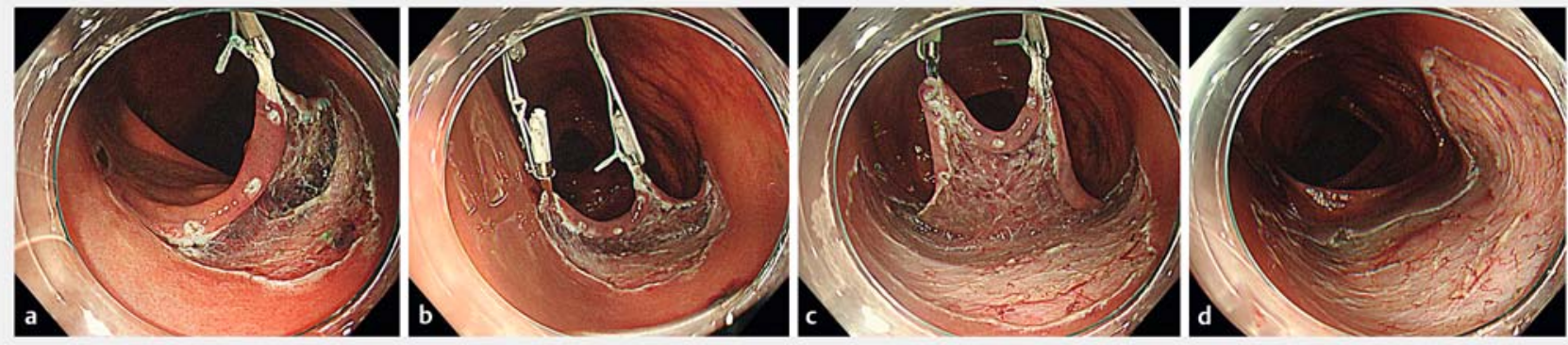

- Fig. 4 Addition of additional M-loop for improved traction. a An LST $30 \mathrm{~mm}$ in size in transverse colon is first dissected to exfoliate the anal edge of the lesion onto which a first M-loop is attached. $\mathbf{b}$ The traction has weakened for the exfoliated mucosa on the left side so additional M-loop was attached. c, $\mathbf{d}$ Submucosal dissection plane became very clearly visible with the addition of two M-loops. The dissection was completed safely. 
procedure time would be expected to be shorter for the M-loop method, but the difference was insignificant between the two groups. In that regard, the M-loop could have been used more for larger lesions to increase SDS. Further study is warranted to clarify this point.

This retrospective study had several limitations. First, because some cases were excluded from the study due to reasons shown in $\mathbf{F i g . 3}$, there was a potential selection bias. Second, this study was carried out in a single center because the M-loop method, which we invented, has not yet been used in many other facilities. Third, we could not directly compare the Mloop and non-traction methods during the same time period. That is because data collection was for all ESD cases from the beginning of 2018 and the M-loop was invented in late 2017. To overcome these limitations, a prospective study will be required in the future.

\section{Conclusion}

In conclusion, the M-loop method effectively increased SDS compared to non-traction methods of ESD. Interestingly, the effect was most prominent in cases performed by non-experts. Therefore, we strongly believe that the M-loop method is a useful tool for colorectal ESD training.

\section{Competing interests}

The authors declare that they have no conflict of interest.

\section{References}

[1] Saito Y, Fukuzawa M, Matsuda T et al. Clinical outcome of endoscopic submucosal dissection versus endoscopic mucosal resection of large colorectal tumors as determined by curative resection. Surg Endosc 2010; 24: 343-352

[2] Terasaki M, Tanaka S, Oka S et al. Clinical outcomes of endoscopic submucosal dissection and endoscopic mucosal resection for laterally spreading tumors larger than $20 \mathrm{~mm}$. J Gastroenterol Hepatol 2012; 27: $734-740$

[3] Oyama T. Counter traction makes endoscopic submucosal dissection easier. Clin Endosc 2012; 45: 375-378
[4] Uraoka T, Parra-Blanco A, Yahagi N. Colorectal endoscopic submucosal dissection: is it suitable in western countries? J Gastroenterol Hepatol 2013; 28: 406-414

[5] Yamada S, Doyama H, Ota R et al. Impact of the clip and snare method using the prelooping technique for colorectal endoscopic submucosal dissection. Endoscopy 2016; 48: 281-285

[6] Ritsuno H, Sakamoto N, Osada T et al. Prospective clinical trial of traction device-assisted endoscopic submucosal dissection of large superficial colorectal tumors using the S-O clip. Surg Endosc 2014; 28: 3143-3149

[7] Sakamoto H, Hayashi Y, Miura Y et al. Pocket-creation method facilitates endoscopic submucosal dissection of colorectal laterally spreading tumors, non-granular type. Endosc Int Open 2017; 5: E123-E129

[8] Kanamori A, Nakano M, Kondo M et al. Clinical effectiveness of the pocket-creation method for colorectal endoscopic submucosal dissection. Endosc Int Open 2017; 5: E1299-E1305

[9] Sudo G, Tanuma T, Suzuki Y et al. Multiloop method for traction during colorectal endoscopic submucosal dissection. VideoGIE 2019; 4: $11-13$

[10] Suzuki Y, Tanuma T, Nojima M et al. Multiloop as a novel traction method in accelerating colorectal endoscopic submucosal dissection. Gastrointest Endosc 2020; 91: 185-190

[11] Watanabe T, Muro K, Ajioka Y et al. Japanese Society for Cancer of the Colon and Rectum (JSCCR) guidelines 2016 for the treatment of colorectal cancer. Int J Clin Oncol 2018; 23: 1-34

[12] Tanaka S, Kashida H, Saito Y et al. JGES guidelines for colorectal endoscopic submucosal dissection/endoscopic mucosal resection. Dig Endosc 2015; 27: 417-434

[13] Hayashi Y, Miura Y, Yamamoto H. Pocket-creation method for the safe, reliable, and efficient endoscopic submucosal dissection of colorectal lateral spreading tumors. Dig Endosc 2015; 27: 534-535

[14] Matsumoto A, Tanaka S, Oba S et al. Outcome of endoscopic submucosal dissection for colorectal tumors accompanied by fibrosis. Scand J Gastroenterol 2010; 45: 1329-1337

[15] Pimentel-Nunes P, Dinis-Ribeiro M, Ponchon T et al. Endoscopic submucosal dissection: European Society of Gastrointestinal Endoscopy (ESGE) Guideline. Endoscopy 2015; 47: 829-854

[16] Shinozaki S, Hayashi Y, Lefor AK et al. What is the best therapeutic strategy for colonoscopy of colorectal neoplasia? Future perspectives from the East Dig Endoscopy 2016; 28: 289-295

[17] Hori K, Uraoka T, Harada K et al. Predictive factors for technically difficult endoscopic submucosal dissection in the colorectum. Endoscopy 2014; 46: 862-870

[18] Kim EK, Han DS, Ro Y et al. The submucosal fibrosis: what does it mean for colorectal endoscopic submucosal dissection? Intest Res 2016; 14: 358-364 